\title{
Papel do cirurgião bucomaxilofacial nas unidades de terapia intensiva
}

The role of the oral and maxillofacial surgeon in intensive care units

Rafaela Riboli*

Simone Pinheiro Siqueira*

Ferdinando de Conto ${ }^{* *}$

\section{Resumo}

Objetivo: fazer uma revisão da literatura que trata das atividades que o cirurgião bucomaxilofacial realiza em pacientes internados nas unidades de terapia intensiva (UTIs). Revisão de literatura: o zelo com a saúde do paciente em âmbito hospitalar exige que esse trabalho seja realizado por uma equipe multiprofissional, o que demandou a inserção da odontologia nesse ambiente. O ganho de espaço nessas equipes ocorreu, principalmente, devido a estudos epidemiológicos, efetuados ao longo dos anos, que mostraram a correlação entre a saúde bucal e a sistêmica. De modo geral, o cirurgião bucomaxilofacial atua no hospital para auxiliar tanto no manejo de pacientes internados em leitos ou em unidades de terapia intensiva quanto para realizar procedimentos orais contraindicados em consultórios odontológicos convencionais. Considerações finais: os pacientes internados nas UTIs estão com seu estado clínico comprometido, estando mais susceptíveis a infecções, por esse motivo, devem receber cuidado odontológico constante como parte integrante da atenção para assegurar a saúde geral.

Palavras-chave: Cirurgia maxilofacial. Odontologia. Unidade de terapia intensiva.

\section{Introdução}

O cirurgião-dentista integra um quadro multidisciplinar no âmbito hospitalar, do qual fazem parte médicos, fonoaudiólogos, nutricionistas, fisioterapeutas e enfermeiros, cujo objetivo é reestabelecer a saúde coletiva dos pacientes ${ }^{1}$. A odontologia ganhou espaço nessas equipes, principalmente, devido a estudos epidemiológicos, efetuados ao longo dos anos, que mostraram a correlação entre a saúde bucal e a sistêmica ${ }^{1,2}$.

A Odontologia Hospitalar começou seu desenvolvimento na América em meados do século XIX, mas chegou ao Brasil somente em 2004, quando foi criada a Associação Brasileira de Odontologia Hospitalar $^{2}$. A partir daquele ano, o profissional odontólogo ingressa nos hospitais para auxiliar tanto no manejo de pacientes internados que apresentam enfermidades sistêmicas quanto para realizar procedimentos orais contraindicados em consultórios odontológicos convencionais ${ }^{3,4}$.

Os pacientes hospitalizados que necessitam de cuidados constantes - monitorização dos sinais vitais, do estado hemodinâmico e da função respiratória - ingressam na unidade de terapia intensiva $(\mathrm{UTI})^{4}$. Infere-se que esse local aumenta de cinco a dez vezes o risco de contrair infecção ${ }^{1}$, pois esses pacientes estão com o estado clínico comprometido, ou seja, apresentam alterações no sistema imunológico, exposição a procedimentos invasivos e desidratação terapêutica (prática comum para aumentar a função respiratória e cardíaca). Ressalta-se, ainda, que esses pacientes estão suscetíveis à xerostomia, especialmente devido à incapacidade de nutrição, hidratação e respiração ${ }^{5}$.

Acadêmicas de Odontologia da Universidade de Passo Fundo, Passo Fundo, RS, Brasil.

* Professor doutor do Programa de Residência em Cirurgia e Traumatologia Bucomaxilofacial. Universidade de Passo Fundo, Passo Fundo, RS, Brasil. 
O objetivo deste trabalho é fazer uma revisão da literatura sobre as atividades que o cirurgião bucomaxilofacial realiza em pacientes internados na UTI.

\section{Métodos}

Para a pesquisa dos estudos incluídos ou considerados neste trabalho, foi realizada uma estratégia de busca detalhada das fontes bibliográficas nos seguintes bancos de dados: PubMed, Bireme, SciELO e Science Directy. Os termos descritores utilizados para essa busca foram cirurgia maxilofacial, unidade de terapia intensiva, infecção dentária e odontologia.

Constatou-se que há publicações sobre 0 assunto desde o ano de 1996. Foram encontradas 29 publicações, do período entre julho de 2014 e janeiro de 2015. Os critérios de inclusão foram: artigos clínicos e de revisão de literatura que abordavam o tema proposto, sendo considerados estudos em língua inglesa e em língua portuguesa. Os critérios de exclusão foram: artigos com ano de publicação anterior a 2000. Os dados foram analisados, cruzados e debatidos para a realização da redação deste estudo.

\section{Discussão}

Infecções odontogênicas são um risco para os pacientes e um importante problema de saúde ${ }^{4}$. O tratamento incorreto ou tardio do problema geralmente leva a complicações graves, como o comprometimento das vias aéreas, o envolvimento do mediastino e da medula, sepses, perda de visão provocada por trombose do seio cavernoso, entre outras condições que poderiam ser evitadas com o diagnóstico preco$\mathrm{ce}^{6,7}$. A infecção proveniente de focos sépticos dentários pode se propagar ao longo dos espaços musculofaciais profundos, resultando em grave edema na laringe e no pescoço. A angina de Ludwig e a celulite cervical oferecem risco de morte. Essas condições exigem, muitas vezes, que o paciente seja submetido à internação sob cuidado constante na UTI ${ }^{7,8}$.

A admissão na UTI do enfermo que apresenta envolvimento infeccioso grave no complexo maxilofacial deve-se à decisão do cirurgião bucomaxilofacial e da equipe médica. É avaliada a necessidade de monitoramento das alterações no estado clínico do paciente bem como a falta de resposta ao tratamento convencional ${ }^{7}$. Cabe ao cirurgião bucomaxilofacial o tratamento da infecção, que consiste, principalmente, na drenagem cirúrgica, sob anestesia geral, na eliminação do foco etiológico e na administração intravenosa de antibióticos ${ }^{6,7}$.

Pesquisadores realizaram um estudo em um hospital público nos Estados Unidos da América que analisou o quadro clínico, o tratamento e o tempo de internação de 42 pacientes internados na UTI por infecção odontogênica. A maior parte, $74 \%$ das infecções, resultou no envolvimento de múltiplos espaços faciais, e a mandíbula foi mais frequentemente envolvida do que a maxila. Foi aplicada, como principal medida terapêutica, a drenagem cirúrgica e o tempo médio de permanência na UTI foi de 3,1 dias. Esse estudo ressaltou que estratégias para melhorar a saúde bucal com a redução da incidência de cárie dentária não tratada reduziriam a porcentagem de pacientes que chegam ao estágio de infecção grave, necessitando, portanto, de internação em centros de terapia intensiva ${ }^{8}$.

Durante o tempo que os pacientes estão internados nas UTIs, eles estão impossibilitados de realizar higiene oral ${ }^{5}$. Possivelmente pela deficiência do relacionamento dos profissionais da odontologia com os demais das equipes da UTI, a condição oral desses pacientes torna-se crítica, o que desencadeia problemas locais, como gengivites, periodontites e cáries, até o comprometimento sistêmico, como otites, faringites e sinusites. O acúmulo de biofilme nas superfícies dentárias associado à redução do fluxo salivar - causado pelo uso de coquetéis medicamentosos - aumenta o risco de doenças, como as pneumonias de aspiração, uma vez que as bactérias presentes na boca podem ser aspiradas, contaminando a árvore respiratória ${ }^{9-11}$.

Além disso, os pacientes que estão em UTIs frequentemente permanecem com a boca aberta devido à intubação traqueal, o que favorece a desidratação da mucosa bucal. A redução do fluxo salivar contribui para o aumento da saburra lingual, propiciando a produção de compostos sulfurados voláteis, tais como mercaptanas e sulfidretos, que causam um odor acentuado bem como a formação de colônias bacterianas organizadas, multiplicando o risco de infecções pulmonares por aspiração $0^{4,5,10,12}$.

Um grupo de pesquisadores acompanhou 73 pacientes com pneumonia nosocomial internados nas UTIs adulto, pediátrica e de queimados do Hospital Metropolitano de Urgência e Emergência, em Belém, PA, com objetivo de avaliar o perfil desses pacientes e os principais agentes infecciosos envolvidos na infecção respiratória. Verificou-se idade média de 38,7 anos, sendo $87,7 \%$ do gênero masculino. A partir do lavado broncoalveolar, da hemocultura e da secreção traqueal, foram obtidas culturas positivas, dezessete tipos diferentes de bactérias foram isolados, sendo Staphylococcus aureus o agente infeccioso mais frequente $(30,7 \%)$. Considerando que esses patógenos estão presentes no meio bucal, pode-se ressaltar que os cuidados odontológicos em pacientes sob terapia intensiva são essenciais, como parte integrante da saúde geral, evitando assim agravos sistêmicos ${ }^{13}$.

Em pacientes internados vítimas de acidentes domésticos ou automobilísticos, é competência do cirurgião bucomaxilofacial avaliar os traumas de face, as lacerações na região maxilofacial, o sangramento e o consequente comprometimento das vias aéreas superiores. Ele precisa estar apto a reconhecer os 
sinais da obstrução e ser hábil em procedimentos cirúrgicos de emergência das vias aéreas, envolvendo-se na equipe multiprofissional de trauma ${ }^{6,14}$.

$\mathrm{O}$ comprometimento das vias aéreas imediato e tardio em trauma facial pode surgir como resultado de combinações variadas de deslocamento de tecido, edema e sangramento, corpos estranhos, vômito e lesão traumática cerebral ${ }^{6}$. Algumas manobras que o cirurgião bucomaxilofacial realiza, como a redução de fraturas deslocadas em traumas maxilofaciais, podem ajudar a reduzir o sangramento e a restaurar parcialmente as vias aéreas ${ }^{14,15}$. As fraturas faciais frequentemente estão associadas a lesões em outras regiões do corpo humano. $\mathrm{O}$ atendimento hospitalar e a assistência ao paciente politraumatizado envolvem uma série de etapas multidisciplinares, capazes de influenciar diretamente a sobrevivência da vítima ${ }^{16}$.

A decisão pelo tratamento cirúrgico é responsabilidade do cirurgião bucomaxilofacial e de sua equipe, porém, quando se tratam de pacientes politraumatizados, essa responsabilidade poderá ser dividida com outros especialistas, em decorrência das outras lesões associadas ${ }^{16}$. Os traumatismos maxilofaciais, quando não estão associados à obstrução das vias aéreas ou a sangramentos importantes, só devem ser tratados após a completa estabilização do paciente e quando as lesões que trazem risco de vida estiverem totalmente controladas ${ }^{15-17}$.

Pacientes com doenças neurológicas, como paralisia cerebral, quando ingressam em um hospital, são mais susceptíveis a permanecer em centros de terapia intensiva, pois necessitam de cuidados constantes. As principais características clínicas que justificam o cuidado ininterrupto são as deficiências ou os distúrbios de movimento e de tônus muscular, os movimentos involuntários e os reflexos anormais, que acabam comprometendo a biomecânica global do indivíduo. No complexo maxilofacial, notam-se transtornos motores bucofonatórios, de modo a apresentar alterações na fala, na mastigação, na deglutição e no controle da saliva, além de traumas, lacerações e disfunções respiratórias ${ }^{18,19}$.

Nesses casos, o cirurgião bucomaxilofacial deve intervir não só nos cuidados com a higiene oral, que nesses pacientes é crítica, mas também deve solucionar complicações advindas dos reflexos espásticos causados pela lesão encefálica ${ }^{18}$. Dentre os distúrbios, o trauma na mucosa bucal e na língua, proveniente da maloclusão ou de mordiscadas, é o mais frequente ${ }^{18,19}$.

Um estudo foi realizado para verificar a eficácia do laser de baixa intensidade utilizado em pacientes com traumatismo de lábio por reflexos espásticos. Após catorze dias da aplicação, a avaliação mostrou que houve redução quase completa das áreas ulceradas e da dor. Assim, pode-se concluir que o cirurgião pode utilizar o laser como ferramenta auxiliar no tratamento desses pacientes. Entretanto, eliminar ou minimizar o fator causador das lesões é pri- mário para a cura total, por isso, em muitos casos, a exodontia é a única alternativa para restauração da saúde oral ${ }^{20}$.

O cirurgião bucomaxilofacial também atua no reconhecimento de potenciais focos sépticos em pacientes internados nas UTIs acometidos com doenças sistêmicas, como a diabete, pois essa situação contribui para a piora clínica do paciente. O problema bucal mais prevalente encontrado nos diabéticos é a doença periodontal. Diversos mecanismos foram propostos para explicar o aumento da susceptibilidade à doença periodontal, tais como: alterações na resposta imune, na microbiota subgengival e no metabolismo do colágeno. No entanto, a infecção periodontal pode agravar o controle glicêmico, elevar o nível de citocinas pró-inflamatórias, causar bacteremia e, consequentemente, aumentar o risco de complicações cardiovasculare ${ }^{21,22}$.

Pesquisadores acompanharam pacientes com diabetes tipo 2 (não insulinodependentes) e relataram que a periodontite grave foi associada à piora do controle glicêmico ao longo do tempo. Indivíduos com periodontite grave, no exame inicial, tiveram maior piora do controle glicêmico ao longo de um período de dois a quatro anos do que aqueles sem periodontite no exame inicial. Nesse estudo, concluiu-se que pacientes com diabetes devem receber avaliação odontológica frequente, para que, se necessário, o tratamento periodontal básico seja realizado ou que os dentes comprometidos sejam removidos, evitando complicações sistêmicas ao enfermo ${ }^{23}$.

$\mathrm{O}$ atendimento hospitalar e a assistência ao paciente internado nos centros de terapia intensiva envolvem uma série de etapas multidisciplinares, capazes de influenciar diretamente a sobrevivência do indivíduo. $\mathrm{O}$ atendimento é realizado em etapas, começando pela avaliação inicial, geralmente feita por um médico cirurgião geral, seguida da avaliação secundária, quando especialistas de cada área são solicitados a avaliar o paciente. Por esse motivo, é importante que o cirurgião bucomaxilofacial conheça o local onde atua, para tanto ele deve ter informações da estrutura funcional e organizacional do hospital e de sua logística. Isso evita perda de tempo durante $o$ atendimento ao enfermo bem como contribui para a diminuição dos custos do atendimento $^{1,2,5,6,16}$.

\section{Considerações finais}

O cirurgião bucomaxilofacial deve conhecer a rotina especial e a complexidade da unidade de terapia intensiva para dar suporte à resolução de traumas maxilofaciais, à liberação de vias aéreas superiores, à detecção e ao tratamento de focos sépticos dentários e ao atendimento a pacientes portadores de necessidades especiais, atuando em cooperação com a equipe multidisciplinar, garantindo 0 suporte de vida ao paciente. 


\section{Abstract}

Objective: This study aimed to perform a literature review on the activities that oral and maxillofacial surgeons perform in patients admitted to intensive care units (ICUs). Literature review: The care with the health of patients in a hospital environment requires the work to be performed by a multidisciplinary team, which demanded the insertion of Dentistry in this environment. The space gained in such teams was mainly due to epidemiological studies conducted over the years, which have shown the correlation between oral and systemic health. Generally, the oral and maxillofacial surgeon works in the hospital to help treating patients admitted to either hospital beds or intensive care units, and to perform oral procedures contraindicated in conventional dental offices. Final considerations: Patients admitted to ICUs are more susceptible to infections, and therefore deserve constant dental care as an integral part of ensuring general health.

Keywords: Maxillofacial surgery. Dentistry. Intensive care units.

\section{Referências}

1. Godoy APT, Francesco AR, Duarte A, Kemp APT, Silva-Lovato $\mathrm{CH}$. Odontologia hospitalar no Brasil: uma visão geral. Rev Odontol Unesp 2009; 38(2):105-9.

2. Aranega AM, Bassi APF, Ponzoni D, Wayama MT, Esteves JC, Garcia Junior IR. Qual a importância da odontologia hospitalar? Rev Bras Odontol 2012; 69(1):90-3.

3. Associação Brasileira de Odontologia [homepage na internet]. [citado 2015 jan. 22]. Disponível em URL: http://www. cbrohi.org.br/projeto-dentista-na-uti-apresenta-mudanca-para-o-mercado.

4. Gaetti-Jardim E, Setti JS, Cheade MFM, Mendonça JGM. Atenção odontológica a pacientes hospitalizados: revisão da literatura e proposta de protocolo de higiene oral. Rev Bras Ciênc Saúde 2013; 35:31-6.

5. Gomes SF, Esteves MCL. Atuação do cirurgião-dentista na UTI: um novo paradigma. Rev Bras Odontol 2012; 69(1):67-70.

6. Perry M, Morris C. Advanced trauma life support (atls) and facial trauma: can one size fit all? Int J Oral Maxillofac Surg 2008; 37:309-20.

7. Ylijoki S, Suuronen R, Jousimies-Somer H, Meurman JH, Lindqvist C. Differences between patients with or without the need for intensive care due to severe odontogenic infections. J Oral Maxillofac Surg 2001; 59:867-72.

8. Jundt JS, Gutta R. Characteristics and cost impact of severe odontogenic infections. J Oral Maxillofac Surg 2012; 114(5):558-66.

9. Amaral SM, Cortês AQ, Pires FR. Pneumonia nosocomial: importância do microambiente oral. J Bras Pneumol 2009; 35(11):1116-24.

10. Beraldo C, Andrade D. Higiene oral com clorexidina na prevenção de pneumonia associada à ventilação mecânica. J Bras Pneumol 2008; 34(9):707-14.

11. Morais TMN, Silva A, Avi ALO, Souza PHR, Knobel E, Camargo LFA. A importância da atuação odontológica em pacientes internados em unidade de terapia intensiva. Rev Bras Ter Intensiva 2006; 18(4):412-7.
12. Pasetti LA, Carneiro Leão MT, Araki LT, Albuquerque AMM, Ramos TMB, Santos SF, et al. Odontologia hospitalar: a importância do cirurgião dentista na unidade de terapia intensiva. Rev Odontol Bauru 2013; 13(4):211-26.

13. Barbosa JCS, Lobato OS, Menezes SAF, Menezes TOA, Pinheiro HHC. Perfil dos pacientes sob terapia intensiva com pneumonia nosocomial: principais agentes etiológicos. Rev Odontol Unesp 2010; 39(4):201-6.

14. Perry M, Dancey A, Mireskandari K, Oakley P, Davies S, Cameron M. Emergency care in facial trauma - a maxillofacial and ophthalmic perspective. Int J Care Injured 2005; 36(5):875-96.

15. Krausz AA, El-Naaj IA, Barak M. Maxillofacial trauma patient: coping with the difficult airway. World J Emerg Surg 2009; 21(4):1-7.

16. Carvalho MF, Herrero RKR, Moreira DR, Urbano ES, Reher P. Princípios de atendimento hospitalar em cirurgia buco-maxilo-facial. Rev Cir Traumatol Buco-maxilo-fac 2010; 10(4):79-84.

17. Scheyerer MJ, Döring R, Fuchs N, Metzler P, Sprengel K, Werner CML, et al. Maxillofacial injuries in severely injured patients. J Trauma Manag Outcomes 2015; 9(4):1-9.

18. Claudino KA, Silva LVC. Complicações respiratórias em pacientes com encefalopatia crônica não progressiva. Rev Neurocienc 2012; 20:94-100.

19. Dougherty NJ. A review of cerebral palsy for the oral health professional. Dental Clinics Of North America 2009; 53(2):329-38.

20. Moreira LA, Santos MTBR, Campos VF, Genovese WF. Efficiency of laser therapy applied in labial traumatism of patients with spastic cerebral palsy. Braz Dent J 2004; 15:29-33.

21. Carneiro Neto JN, Beltrame M, Souza IFA, Andrade JM, Silva JAL, Quintela KL. O paciente diabético e suas implicações para conduta odontológica. Rev Dentística 2012; 11(23):11-8.

22. Yamashita JM, Moura-Grec PG, Capelari MM, Sales-Peres A, Sales-Peres SHC. Manifestações bucais em pacientes portadores de diabetes mellitus: uma revisão sistemática. Rev Odontol Unesp 2013; 42(3):211-20.

23. Schara R, Medvesck M, Skaleric U. Periodontal disease and diabetes metabolic control: a full-mouth disinfection approach. J Int Acad Periodontol 2006; 8:61-6.

\section{Endereço para correspondência:}

Rafaela Riboli

Rua Cai, 92 Bairro Boqueirão

99010-000 Passo Fundo, RS, Brasil

Telefone: (54) 99984-2240

E-mail: rafa_riboli@hotmail.com

Recebido: 29/03/2016. Aceito: 02/06/2016. 